\title{
ON STRUCTURED VARIANTS OF MODIFIED HSS ITERATION METHODS FOR COMPLEX TOEPLITZ LINEAR SYSTEMS*
}

\author{
Fang Chen \\ Department of Mathematical Sciences, Xi'an Jiaotong University, Xi'an 710049, China \\ Key Laboratory of Environmental Mechanics, Institute of Mechanics, Chinese Academy of Sciences, \\ Beijing 100190, China \\ Email: chenfreesky@gmail.com \\ Yaolin Jiang \\ Department of Mathematical Sciences, Xi'an Jiaotong University, Xi'an 710049, China \\ Email: yljiang@mail.xjtu.edu.cn \\ Qingquan Liu \\ Key Laboratory of Environmental Mechanics, Institute of Mechanics, Chinese Academy of Sciences, \\ Beijing 100190, China \\ Email: qqliu@imech.ac.cn
}

\begin{abstract}
The Modified Hermitian and skew-Hermitian splitting (MHSS) iteration method was presented and studied by Bai, Benzi and Chen (Computing, 87(2010), 93-111) for solving a class of complex symmetric linear systems. In this paper, using the properties of Toeplitz matrix, we propose a class of structured MHSS iteration methods for solving the complex Toeplitz linear system. Theoretical analysis shows that the structured MHSS iteration method is unconditionally convergent to the exact solution. When the MHSS iteration method is used directly to complex symmetric Toeplitz linear systems, the computational costs can be considerately reduced by use of Toeplitz structure. Finally, numerical experiments show that the structured MHSS iteration method and the structured MHSS preconditioner are efficient for solving the complex Toeplitz linear system.
\end{abstract}

Mathematics subject classification: 65F10, 65F50.

Key words: Toeplitz matrix, MHSS iteration method, Complex symmetric linear system.

\section{Introduction}

We consider the complex Toeplitz linear system

$$
A x=b,
$$

with $A \in \mathbb{C}^{n \times n}$ a large, non-Hermitian, and positive definite Toeplitz matrix, and $x, b \in \mathbb{C}^{n}$. The complex Toeplitz matrices arise in solutions of differential and integral equations, and in some practical problems and mathematical methods in physics, statistics, and signal processing.

\footnotetext{
* Received January 15, 2012 / Revised version received April 4, 2012 / Accepted August 7, 2012 /

Published online January 17, 2013 /
} 
A complex Toeplitz matrix $A$ has the form

$$
A=\left(\begin{array}{ccccc}
a_{0} & a_{1} & a_{2} & \cdots & a_{n-1} \\
a_{-1} & \ddots & \ddots & \ddots & \vdots \\
a_{-2} & \ddots & \ddots & \ddots & a_{2} \\
\vdots & \ddots & \ddots & \ddots & a_{1} \\
a_{-n+1} & \ddots & a_{-2} & a_{-1} & a_{0}
\end{array}\right),
$$

where $a_{j} \in \mathbb{C}$. From (1.2) it is easy to know that the matrix $A$ is decided by the elements of its first row and first column. To solve the complex Toeplitz linear system (1.1), actual structure and efficient splitting of the Toeplitz matrix $A$ are important and need to be discussed in depth.

Recently, the Hermitian and skew-Hermitian splitting (HSS) iteration method has been paid great attention. Bai, Golub and Ng introduced the HSS iteration method for solving the nonHermitian positive definite linear systems in [8]. Based on the HSS iteration method, Bai, Benzi and Chen established the modified HSS (MHSS) iteration method for solving a class of important complex symmetric linear systems in [4], and proposed the generalized MHSS (GMHSS) iteration method, too. According to properties of the saddle point matrix, Bai and Golub proposed the accelerated HSS (AHSS) iteration method in [5]. In addition, Gu and Tian used the HSS iteration method to solve the Toeplitz linear system in [13], and Chen and Jiang presented the structured HSS and the structured AHSS iteration methods for solving the real Toeplitz linear systems in [11]; see also [1,2,6,7,9,14,15].

We now describe the HSS, MHSS and GMHSS iteration methods. To this end, the matrix $A$ is split into its Hermitian and skew-Hermitian parts as

$$
A=H+S,
$$

where $H=\frac{1}{2}\left(A+A^{*}\right)$ and $S=\frac{1}{2}\left(A-A^{*}\right)$; see [3]. Here, $A^{*}$ is used to denote the conjugate transpose of the matrix $A$. Based on this splitting, the HSS iteration method [8] can be described as follows.

The HSS Iteration Method. Given an initial guess $x^{(0)} \in \mathbb{C}^{n}$, for $k=0,1,2, \ldots$ until the iteration sequence $\left\{x^{(k)}\right\}$ converges, compute $x^{(k+1)}$ using the following procedure:

$$
\left\{\begin{array}{l}
(\alpha I+H) x^{\left(k+\frac{1}{2}\right)}=(\alpha I-S) x^{(k)}+b, \\
(\alpha I+S) x^{(k+1)}=(\alpha I-H) x^{\left(k+\frac{1}{2}\right)}+b,
\end{array}\right.
$$

where $\alpha$ is a given positive constant, and $I$ is the identity matrix.

When the coefficient matrix $A$ is complex symmetric, we have

$$
A=\operatorname{Re}(A)+\imath \operatorname{Im}(A),
$$

where $\operatorname{Re}(A)$ and $\operatorname{Im}(A)$ denote the real and the imaginary parts of the matrix $A$, respectively, and $\imath$ represents the imaginary unit. For the complex symmetric linear system, the MHSS iteration method [4] is described as follows.

The MHSS Iteration Method. Given an initial guess $x^{(0)} \in \mathbb{C}^{n}$, for $k=0,1,2, \ldots$ until the iteration sequence $\left\{x^{(k)}\right\}$ converges, compute $x^{(k+1)}$ using the following procedure:

$$
\left\{\begin{aligned}
(\alpha I+\operatorname{Re}(A)) x^{\left(k+\frac{1}{2}\right)} & =(\alpha I-\operatorname{Im}(A) \imath) x^{(k)}+b, \\
(\alpha I+\operatorname{Im}(A)) x^{(k+1)} & =(\alpha I+\operatorname{Re}(A) \imath) x^{\left(k+\frac{1}{2}\right)}-\imath b,
\end{aligned}\right.
$$


where $\alpha$ is a given positive constant.

Analogous to the HSS splitting, the complex matrix $A$ has the splitting

$$
A=W+\imath T,
$$

where

$$
W=\frac{1}{2}\left(A+A^{*}\right), \quad T=\frac{1}{2 \imath}\left(A-A^{*}\right) .
$$

According to this splitting, the GMHSS iteration method [4] is described as follows.

The GMHSS Iteration Method. Given an initial guess $x^{(0)} \in \mathbb{C}^{n}$, for $k=0,1,2, \ldots$ until the iteration sequence $\left\{x^{(k)}\right\}$ converges, compute $x^{(k+1)}$ using the following procedure:

$$
\left\{\begin{array}{l}
(\alpha I+W) x^{\left(k+\frac{1}{2}\right)}=(\alpha I-\imath T) x^{(k)}+b, \\
(\alpha I+T) x^{(k+1)}=(\alpha I+\imath W) x^{\left(k+\frac{1}{2}\right)}-\imath b,
\end{array}\right.
$$

where $\alpha$ is a given positive constant.

The HSS, MHSS, and GMHSS iteration methods have been proved to be convergent to the unique solution of the system of linear equations $A x=b$ where the matrix $A \in \mathbb{C}^{n \times n}$ is positive definite.

In this paper, the MHSS iteration method is further specilized to solve the complex Toeplitz linear system (1.1). Because a complex Toeplitz linear system can be transformed into a complex symmetric linear system, we propose the structured MHSS iteration method for solving the complex Toeplitz linear system (1.1). In particular, when the real and imaginary parts of the Toeplitz matrix is symmetric, we show that the sizes of the sub-systems of linear equations involved in the two-half steps of the MHSS iteration method can be considerably reduced. This method is called the MHSS-based structured iteration method, and it saves time when used to solve the Toeplitz linear system.

The remainder of the paper is organized as follows: In Section 2 the complex Toeplitz linear system is converted to a complex symmetric linear system. The structured MHSS iteration method is established and analyzed in Section 3. In Section 4, the symmetric Toeplitz linear systems are discussed in detail, and the MHSS-based structured iteration method is established. Finally, in Section 5, numerical examples are used to examine the effectiveness of the structured MHSS and the MHSS-based structured iteration methods.

\section{Some Properties of Complex Toeplitz Matrices}

In this section, a complex Toeplitz linear system is transformed into a complex symmetric linear system by employing a unitary matrix.

Let the complex Toeplitz matrix $A$ have the form (1.2). Then its Hermitian part $H$ is a centrohermitian matrix and its skew-Hermitian part $S$ is a skew-centrohermitian matrix. Equivalently, $H$ and $S$ satisfy

$$
\bar{H}=J_{n} H J_{n}, \quad \bar{S}=-J_{n} S J_{n},
$$

where $J_{n} \in \mathbb{C}^{n \times n}$ is a permutation matrix with ones on the cross diagonal (bottom left to top right) and zeros elsewhere, we use $\bar{H}$ and $\bar{S}$ to denote the conjugate matrices of $H$ and $S$, respectively. 
According to the cases that $n$ is even and odd, the structures of the matrices $H$ and $S$ are outlined in the following.

(a) When $n=2 m$, the matrix $H$ can be partitioned into the form

$$
H=\left(\begin{array}{cc}
B & C^{*} \\
C & B
\end{array}\right)
$$

where the top row of the Hermitian Toeplitz matrix $B$ is

$$
\frac{1}{2}\left[a_{0}+\bar{a}_{0}, a_{1}+\bar{a}_{-1}, \ldots, a_{m-1}+\bar{a}_{-m+1}\right],
$$

and the first row and the first column of the Toeplitz matrix $C$ are

$$
\frac{1}{2}\left[a_{-m}+\bar{a}_{m}, a_{-m+1}+\bar{a}_{m-1}, \ldots, a_{-1}+\bar{a}_{1}\right]
$$

and

$$
\frac{1}{2}\left[a_{-m}+\bar{a}_{m}, a_{-m-1}+\bar{a}_{m+1}, \ldots, a_{-n+1}+\bar{a}_{n-1}\right]^{T},
$$

respectively. Similarly, the matrix $S$ can be written as the form

$$
S=\left(\begin{array}{cc}
D & -E^{*} \\
E & D
\end{array}\right)
$$

where the top row of the skew-Hermitian Toeplitz matrix $D$ is

$$
\frac{1}{2}\left[a_{0}-\bar{a}_{0}, a_{1}-\bar{a}_{-1}, \ldots, a_{m-1}-\bar{a}_{-m+1}\right],
$$

the top row and the first column of the Toeplitz matrix $E$ are

$$
\frac{1}{2}\left[a_{-m}-\bar{a}_{m}, a_{-m+1}-\bar{a}_{m-1}, \ldots, a_{-1}-\bar{a}_{1}\right]
$$

and

$$
\frac{1}{2}\left[a_{-m}-\bar{a}_{m}, a_{-m-1}-\bar{a}_{m+1}, \ldots, a_{-n+1}-\bar{a}_{n-1}\right]^{T},
$$

respectively. By using the unitary matrix

$$
Q=\frac{\sqrt{2}}{2}\left(\begin{array}{cc}
I_{m} & \imath I_{m} \\
J_{m} & -\imath J_{m}
\end{array}\right),
$$

we have the following equalities:

$$
\begin{gathered}
Q^{*} H Q=\left(\begin{array}{cc}
\operatorname{Re}\left(B+J_{m} C\right) & -\operatorname{Im}\left(B+J_{m} C\right) \\
\operatorname{Im}\left(B-J_{m} C\right) & \operatorname{Re}\left(B-J_{m} C\right)
\end{array}\right):=\left[\begin{array}{cc}
\hat{H}_{11} & \hat{H}_{12} \\
\hat{H}_{12} & \hat{H}_{22}
\end{array}\right]=\hat{H}, \\
Q^{*} S Q=\imath\left(\begin{array}{cc}
\operatorname{Im}\left(D+J_{m} E\right) & \operatorname{Re}\left(D+J_{m} E\right) \\
-\operatorname{Re}\left(D-J_{m} E\right) & \operatorname{Im}\left(D-J_{m} E\right)
\end{array}\right):=\imath\left[\begin{array}{cc}
\hat{S}_{11} & \hat{S}_{12} \\
\hat{S}_{12} & \hat{S}_{22}
\end{array}\right]=\imath \hat{S} .
\end{gathered}
$$


Evidently, $\hat{H}$ and $\hat{S}$ are real matrices satisfying $\hat{H}^{T}=\hat{H}$ and $\hat{S}^{T}=\hat{S}$, as

$$
-\left[\operatorname{Im}\left(B+J_{m} C\right)\right]^{T}=\operatorname{Im}\left(B-J_{m} C\right), \quad\left[\operatorname{Re}\left(D+J_{m} E\right)\right]^{T}=-\operatorname{Re}\left(D-J_{m} E\right) .
$$

Let

$$
\hat{A}=Q^{*} A Q, \quad \hat{x}=Q^{*} x, \quad \hat{b}=Q^{*} b .
$$

Then the linear system (1.1) is equivalent to

$$
\hat{A} \hat{x}=\hat{b}
$$

From the above analysis, we observe that

$$
\operatorname{Re}(\hat{A})=\hat{H}, \quad \operatorname{Im}(\hat{A})=\hat{S},
$$

and the matrix $\hat{A}$ possesses the Hermitian and skew-Hermitian splitting

$$
\hat{A}=\operatorname{Re}(\hat{A})+\imath \operatorname{Im}(\hat{A}) .
$$

Moreover, the real and the imaginary parts of $\hat{A}$ are real symmetric. Note that

$$
\begin{aligned}
\hat{A} & =\left(\begin{array}{ll}
\operatorname{Re}\left(B+J_{m} C\right)+\imath \operatorname{Im}\left(D+J_{m} E\right) & -\operatorname{Im}\left(B+J_{m} C\right)+\imath \operatorname{Re}\left(D+J_{m} E\right) \\
\operatorname{Im}\left(B-J_{m} C\right)-\imath \operatorname{Re}\left(D-J_{m} E\right) & \operatorname{Re}\left(B-J_{m} C\right)+\imath \operatorname{Im}\left(D-J_{m} E\right)
\end{array}\right) \\
& :=\left(\begin{array}{cc}
\hat{A}_{11} & \hat{A}_{12} \\
\hat{A}_{21} & \hat{A}_{22}
\end{array}\right),
\end{aligned}
$$

with

$$
\hat{A}_{11}^{T}=\hat{A}_{11}, \quad \hat{A}_{22}^{T}=\hat{A}_{22}, \quad \hat{A}_{12}^{T}=\hat{A}_{21} .
$$

Then the linear system $(2.2)$ has a complex symmetric coefficient matrix $\hat{A}$.

(b) When $n=2 m+1$, the matrices $H$ and $S$ can be partitioned into the following forms:

$$
H=\left(\begin{array}{ccc}
B & J_{m} \bar{r} & C^{*} \\
r^{T} J_{m} & \operatorname{Re}\left(a_{0}\right) & r^{*} \\
C & r & B
\end{array}\right)
$$

and

$$
S=\left(\begin{array}{ccc}
D & -J_{m} \bar{z} & -E^{*} \\
z^{T} J_{m} & \imath \operatorname{Im}\left(a_{0}\right) & -z^{*} \\
E & z & D
\end{array}\right)
$$

Define a unitary matrix

$$
Q=\frac{\sqrt{2}}{2}\left(\begin{array}{ccc}
I_{m} & 0 & \imath I_{m} \\
0 & \sqrt{2} & 0 \\
J_{m} & 0 & -\imath J_{m}
\end{array}\right)
$$

Then it holds that

$$
Q^{*} H Q=\left(\begin{array}{ccc}
\operatorname{Re}\left(B+J_{m} C\right) & \sqrt{2} J_{m} \operatorname{Re}(r) & -\operatorname{Im}\left(B+J_{m} C\right) \\
\sqrt{2} \operatorname{Re}\left(r^{T}\right) J_{m} & \operatorname{Re}\left(a_{0}\right) & -\sqrt{2} \operatorname{Im}\left(r^{T}\right) J_{m} \\
\operatorname{Im}\left(B-J_{m} C\right) & -\sqrt{2} J_{m} \operatorname{Im}(r) & \operatorname{Re}\left(B-J_{m} C\right)
\end{array}\right):=\tilde{H}
$$


and

$$
Q^{*} S Q=\imath\left(\begin{array}{ccc}
\operatorname{Im}\left(D+J_{m} E\right) & \sqrt{2} J_{m} \operatorname{Im}(z) & \operatorname{Re}\left(D+J_{m} E\right) \\
\sqrt{2} \operatorname{Im}\left(z^{T}\right) J_{m} & \operatorname{Im}\left(a_{0}\right) & \sqrt{2} \operatorname{Rm}\left(z^{T}\right) J_{m} \\
-\operatorname{Re}\left(D-J_{m} E\right) & \sqrt{2} J_{m} \operatorname{Re}(z) & \operatorname{Im}\left(D-J_{m} E\right)
\end{array}\right):=\imath \tilde{S}
$$

Hence, a linear system analogous to $(2.2)$ can be obtained, too.

\section{The Structured MHSS Iteration Method}

In this section, a structured MHSS iteration method for solving the linear system (1.1) is proposed based on the MHSS iteration method. For convenience, we only describe the case of $n=2 m$, as the case $n=2 m+1$ can be treated in an analogous fashion. Based on the real and the imaginary parts, the matrix $\hat{A}$ can be written as

$$
\hat{A}=\operatorname{Re}(\hat{A})+\imath \operatorname{Im}(\hat{A}) .
$$

We assume that $\operatorname{Re}(\hat{A})$ is positive definite and $\operatorname{Im}(\hat{A})$ is positive semidefinite, respectively.

In fact, after transforming the Toeplitz linear system into the complex symmetric linear system, we use MHSS iteration method to solve the linear system (2.2). This leads to the socalled structured MHSS iteration method for the linear system (1.1), which is algorithmically described as follows.

The Structured MHSS Iteration Method. Given a starting guess $x^{(0)} \in \mathbb{C}^{n}$, let $\hat{x}^{(0)}=Q^{*} x^{(0)}$, compute $\hat{x}^{(k+1)}$ for $k=0,1,2, \ldots$, using the following iteration scheme until $\left\{\hat{x}^{(k)}\right\}$ converges:

Step 1: Compute $\hat{x}^{\left(k+\frac{1}{2}\right)}=\left(\left(\hat{x}_{1}^{\left(k+\frac{1}{2}\right)}\right)^{T},\left(\hat{x}_{2}^{\left(k+\frac{1}{2}\right)}\right)^{T}\right)^{T}$ by

$$
\begin{gathered}
\left(\alpha I+H_{11}-H_{12}\left(\alpha I+H_{22}\right)^{-1} H_{12}\right) \hat{x}_{1}^{\left(k+\frac{1}{2}\right)}=\left(\alpha I-\imath S_{11}+\imath H_{12}\left(\alpha I+H_{22}\right)^{-1} S_{21}\right) \hat{x}_{1}^{(k)} \\
-\left(\imath S_{12}+H_{12}\left(\alpha I+H_{22}\right)^{-1}\left(\alpha I-\imath S_{12}\right)\right) \hat{x}_{2}^{(k)}-H_{12}\left(\alpha I+H_{22}\right)^{-1} \hat{b}_{2}+\hat{b}_{1}, \\
\left(\alpha I+H_{22}\right) \hat{x}_{2}^{\left(k+\frac{1}{2}\right)}=-H_{12} \hat{x}_{1}^{\left(k+\frac{1}{2}\right)}-\imath S_{21} \hat{x}_{1}^{(k)}+\left(\alpha I-\imath S_{22}\right) \hat{x}_{2}^{(k)}+\hat{b}_{2} ;
\end{gathered}
$$

Step 2: Compute $\hat{x}^{(k)}=\left(\left(\hat{x}_{1}^{(k)}\right)^{T},\left(\hat{x}_{2}^{(k)}\right)^{T}\right)^{T}$ by

$$
\begin{gathered}
\left(\alpha I+\imath S_{11}-S_{12}\left(\alpha I+S_{22}\right)^{-1} S_{12}\right) \hat{x}_{1}^{(k+1)}=\left(\alpha I+\imath H_{11}-\imath S_{12}\left(\alpha I+S_{22}\right)^{-1} H_{12}\right) \hat{x}_{1}^{\left(k+\frac{1}{2}\right)} \\
+\left(\imath H_{12}-S_{12}\left(\alpha I+S_{22}\right)^{-1}\left(\alpha I+\imath H_{22}\right)\right) \hat{x}_{2}^{\left(k+\frac{1}{2}\right)}-\imath \hat{b}_{1}+\imath S_{12}\left(\alpha I+S_{22}\right)^{-1} \hat{b}_{2} \\
\left.\left(\alpha I+S_{22}\right)\right) \hat{x}_{2}^{(k+1)}=-S_{12} \hat{x}_{1}^{(k+1)}+\imath H_{12} \hat{x}_{1}^{\left(k+\frac{1}{2}\right)}+\left(\alpha I+\imath H_{22}\right) \hat{x}_{2}^{\left(k+\frac{1}{2}\right)}-\imath \hat{b}_{2}
\end{gathered}
$$

Step 3: Compute $x^{(k+1)}$ by

$$
x^{(k+1)}=Q \hat{x}^{(k+1)}
$$

where $\alpha$ is a given positive constant, $\hat{b}_{i} \in \mathbb{C}^{m}(i=1,2), \hat{b}=\left(\hat{b}_{1}^{T}, \hat{b}_{2}^{T}\right)^{T}$.

Evidently, at each iteration of the structured MHSS iteration method we need to solve two linear sub-systems with coefficient matrices $\alpha I+\operatorname{Re}(\hat{A})$ and $\alpha I+\operatorname{Im}(\hat{A})$. Under our assumptions, both of these matrices are real, symmetric and positive definite for all $\alpha>0$.

According to the MHSS iteration method for the new system (2.2), it leads to the MHSS preconditioner $\hat{B}(\alpha)$, for the matrix $\hat{A}$, here

$$
\hat{B}(\alpha)=\frac{1+\imath}{2 \alpha}(\alpha I+\hat{H})(\alpha I+\hat{S}) .
$$

Now, let us analyze the convergence of the structured MHSS iteration method. 
Theorem 3.1. Let the Toeplitz matrix $A \in \mathbb{C}^{n \times n}$ be positive definite, $H=\frac{1}{2}\left(A+A^{*}\right), S=$ $\frac{1}{2}\left(A-A^{*}\right)$, and $-\imath S$ be positive semidefinite. Then the iteration matrix $M(\alpha)$ of the structured MHSS iteration method is given by

$$
M(\alpha)=(\alpha I-\imath S)^{-1}(\alpha I+\imath H)(\alpha I+H)^{-1}(\alpha I-S) .
$$

The spectral radius $\rho(M(\alpha))$ of $M(\alpha)$ is bounded by

$$
\delta(\alpha)=\max _{\lambda_{i} \in \lambda(H)} \frac{\sqrt{\alpha^{2}+\lambda_{i}^{2}}}{\alpha+\lambda_{i}} .
$$

Therefore, for any given parameter $\alpha>0$, it holds that

$$
\rho(M(\alpha)) \leq \delta(\alpha)<1
$$

i.e., the structured MHSS iteration method converges unconditionally to the unique solution of the system of linear equations (1.1).

Proof. Let

$$
\hat{M}(\alpha)=Q(\alpha I+\operatorname{Im}(\hat{A}))^{-1}(\alpha I+\imath \operatorname{Re}(\hat{A}))(\alpha I+\operatorname{Re}(\hat{A}))^{-1}(\alpha I-\imath \operatorname{Im}(\hat{A})) Q^{*} .
$$

where the matrix $Q$ is defined as in (2.1), and $\hat{A}=Q^{*} A Q$. Then

$$
M(\alpha)=\hat{M}(\alpha)
$$

Assume

$$
\begin{aligned}
& U(\alpha)=(\alpha I+\imath \operatorname{Re}(\hat{A}))(\alpha I+\operatorname{Re}(\hat{A}))^{-1}, \\
& V(\alpha)=(\alpha I-\imath \operatorname{Im}(\hat{A}))(\alpha I+\operatorname{Im}(\hat{A}))^{-1} .
\end{aligned}
$$

Then by using the similarity invariance of the matrix spectrum, we get

$$
\rho(M(\alpha))=\rho(\hat{M}(\alpha)) \leq\|(U(\alpha) V(\alpha))\|_{2} \leq\|U(\alpha)\|_{2}\|V(\alpha)\|_{2} .
$$

Notice that $\operatorname{Re}(\hat{A})$ and $\operatorname{Im}(\hat{A})$ are real symmetric matrices. So the eigenvalues of $\operatorname{Re}(\hat{A})$ and $\operatorname{Im}(\hat{A})$ are real numbers. Hence, we get

$$
\begin{aligned}
\|U(\alpha)\|_{2} & =\max _{\lambda_{i} \in \lambda(\operatorname{Re}(\hat{A}))} \frac{\sqrt{\alpha^{2}+\lambda_{i}^{2}}}{\left|\alpha+\lambda_{i}\right|} \\
\|V(\alpha)\|_{2} & =\max _{\mu_{i} \in \mu(\operatorname{Im}(\hat{A}))} \frac{\sqrt{\alpha^{2}+\mu_{i}^{2}}}{\left|\alpha+\mu_{i}\right|} .
\end{aligned}
$$

Because

$$
\lambda_{i}>0, \mu_{i} \geq 0
$$

we easily see that

$$
\|U(\alpha)\|_{2}<1,\|V(\alpha)\|_{2} \leq 1
$$

Therefore,

$$
\rho(M(\alpha)) \leq\|U(\alpha)\|_{2}\|V(\alpha)\|_{2} \leq\|U(\alpha)\|_{2}=\delta(\alpha)<1 .
$$


This completes the proof of the theorem.

Theorem 3.1 shows that the convergence speed of the structured MHSS iteration method is bounded by $\delta(\alpha)$, which depends on the spectrum of the Hermitian part $H$, but does not depend on the eigenvectors of other matrices involved. In the following, we give the optimal choice for the parameter $\alpha$, which minimizes the upper bound $\delta(\alpha)$.

Corollary 3.1. Let the conditions of Theorem 3.1 be satisfied. Let $\lambda_{\min }$ and $\lambda_{\max }$ be the minimal and the maximal eigenvalues of the Hermitian positive definite matrix $H$, respectively. Then

$$
\alpha_{\star}=\sqrt{\lambda_{\min } \lambda_{\max }}
$$

and

$$
\delta\left(\alpha_{\star}\right)=\frac{\sqrt{\lambda_{\min }+\lambda_{\max }}}{\sqrt{\lambda_{\min }}+\sqrt{\lambda_{\max }}}=\frac{\sqrt{\kappa(H)+1}}{\sqrt{\kappa(H)}+1},
$$

where $\kappa(H)$ is the spectral condition number of the matrix $H$.

Proof. We easily see that

$$
\delta(\alpha)=\max \left\{\frac{\sqrt{\alpha^{2}+\left(\lambda_{\min }\right)^{2}}}{\alpha+\lambda_{\min }}, \frac{\sqrt{\alpha^{2}+\left(\lambda_{\max }\right)^{2}}}{\alpha+\lambda_{\max }}\right\} .
$$

So the minimal value of the function $\delta(\alpha)$ is attained when

$$
\frac{\sqrt{\alpha^{2}+\left(\lambda_{\min }\right)^{2}}}{\alpha+\lambda_{\min }}=\frac{\sqrt{\alpha^{2}+\left(\lambda_{\max }\right)^{2}}}{\alpha+\lambda_{\max }} .
$$

After solving the above equation, we obtain

$$
\alpha_{\star}=\sqrt{\lambda_{\min } \lambda_{\max }}
$$

By substituting $\alpha_{\star}$ into the formula of $\delta(\alpha)$, we get the result.

\section{A Special Case}

In this section, we discuss a kind of special Toeplitz linear system $A x=b$, where $A$ has the form

$$
A=W+\imath T
$$

where $W$ and $T$ are real symmetric Toeplitz matrices, see [4]. We can use the MHSS iteration method (1.3) to solve this kind of linear system. We know that the linear sub-systems with the coefficient matrices $\alpha I+W$ and $\alpha I+T$ need to be solved at each step of the MHSS iteration. Because these two matrices are still real symmetric Toeplitz ones, we can use the method in [11] to solve them. We call it the MHSS-based structured iteration method. As $\alpha I+W$ and $\alpha I+T$ become two-by-two block diagonal matrices with orthogonal transforms, we only need to solve four smaller linear sub-systems in actual implementations. 


\section{Numerical Examples}

In this section, we give numerical examples to illustrate the effectiveness of the structured MHSS iteration methods. In our implementations, all programs are performed in MATLAB with machine precision $10^{-16}$, and terminated when the current residual satisfies

$$
\mathrm{RES}=: \frac{\left\|r^{(k)}\right\|_{2}}{\left\|r^{(0)}\right\|_{2}}<10^{-6}
$$

where $r^{(k)}$ is the residual at the $k$ th iterate. The zero vector is chosen as the initial guess, and the right-hand side vector $b$ is obtained by $b=A l$, with $l$ being the vector of all elements $1+\imath$.

We know that a Toeplitz matrix is often generated by a $2 \pi$-periodic function $f(x)$ in applications. The function and the elements of the Toeplitz matrix have the relationship

$$
a_{k}=\frac{1}{2 \pi} \int_{-\pi}^{\pi} f(x) e^{\imath k x} d x, \quad k=0, \pm 1, \ldots, \pm(n-1),
$$

and $a_{k}$ is called the Fourier coefficients of the function $f(x)$; see [10,12].

The generating function $f(x)$ in $[-\pi, \pi]$ and the spectra of the corresponding matrix have the following relationship:

(a) when $f(x)$ is a real-valued function, the eigenvalues of the matrix $A$ are real and satisfy

$$
\lambda_{\min } \leq \min f(x) \leq \max f(x) \leq \lambda_{\max } ;
$$

(b) when $f(x)$ is a complex-valued function, the eigenvalues of the matrix $A$ are complex and satisfy

$$
\begin{gathered}
\operatorname{Re}(\lambda)_{\min } \leq \min \operatorname{Re}(f(x)) \leq \max \operatorname{Re}(f(x)) \leq \operatorname{Re}(\lambda)_{\max } \\
\operatorname{Im}(\lambda)_{\min } \leq \min \operatorname{Im}(f(x)) \leq \max \operatorname{Im}(f(x)) \leq \operatorname{Im}(\lambda)_{\max }
\end{gathered}
$$

where $\lambda_{\min }$ and $\lambda_{\max }$ denote the minimal and the maximal eigenvalues of the generated Toeplitz matrix $A, \operatorname{Re}(\lambda)_{\text {min }}$ and $\operatorname{Re}(\lambda)_{\max }$ as well as $\operatorname{Im}(\lambda)_{\min }$ and $\operatorname{Im}(\lambda)_{\max }$ represent the minimal and the maximal of the real as well as the imaginary parts of the eigenvalue $\lambda$, respectively.

Example 5.1. The generating function is given by

$$
f(x)=22+x^{2}+x^{3} .
$$

Table 5.1: Results of the Iteration Methods for Example 5.1.

\begin{tabular}{|c|c|c|c|c|c|c|}
\hline & \multicolumn{3}{|c|}{ structured MHSS } & \multicolumn{2}{c|}{ GMRES } \\
\hline$n$ & $\alpha_{\text {opt }}$ & $\alpha^{t}$ & IT & CPU & IT & CPU \\
\hline 48 & {$[19.21,21.27]$} & 20.91 & 42 & 0.002 & 16 & 0.004 \\
\hline 64 & {$[18.50,18.65]$} & 18.54 & 43 & 0.003 & 18 & 0.005 \\
\hline 96 & {$[16.37,17.72]$} & 17.27 & 45 & 0.006 & 20 & 0.005 \\
\hline 128 & {$[15.72,17.07]$} & 15.80 & 46 & 0.010 & 21 & 0.006 \\
\hline 160 & {$[14.95,17.21]$} & 15.93 & 47 & 0.015 & 23 & 0.011 \\
\hline 192 & {$[14.92,17.00]$} & 16.26 & 47 & 0.021 & 23 & 0.009 \\
\hline 384 & {$[15.68,17.46]$} & 16.28 & 45 & 0.124 & 23 & 0.016 \\
\hline
\end{tabular}


In our tests, the experimentally found optimal parameters $\alpha_{\text {opt }}$ are obtained according to the minimal numbers of iteration steps. When these optimal parameters form intervals, we get the experimentally found optimal parameters $\alpha^{t}$ according to the lest computing times. Moreover, we compare these iteration methods in the senses of iteration step (denoted as IT) and CPU time (denotes as CPU).

We use the structured MHSS and the GMRES iteration methods to solve the linear system generated by the function $f(x)$ in Example 5.1. In Table 5.1, we list the experimentally found optimal intervals and optimal parameters $\alpha^{t}$, IT and CPU, with respect to different $n$. In Table 5.2, the results of the structured MHSS and Circulant preconditioned GMRES method are listed. These two iteration methods are abbreviated as SMHSS-GMRES and Cir-GMRES, respectively.

The numerical results in Tables 5.1 and 5.2 show that the structured MHSS iteration method cost more iteration steps and computing time than the GMRES method, but as preconditioners, the SMHSS-GMRES method outperform the Cir-GMRES method [10] in CPU time and iteration step. Form Table 5.2, we know that IT and CPU of the SMHSS-GMRES and the Cir-GMRES methods almost have no change with respect to different $n$.

Table 5.2: Results of the SMHSS-GMRES and Cir-GMRES Methods for Example 5.1.

\begin{tabular}{|c|c|c|c|c|c|c|}
\hline & \multicolumn{4}{|c|}{ SMHSS-GMRES } & \multicolumn{2}{c|}{ Cir-GMRES } \\
\hline$n$ & $\alpha_{\text {opt }}$ & $\alpha^{t}$ & IT & CPU & IT & CPU \\
\hline 48 & {$[0.01,0.09]$} & 0.07 & 2 & 0.001 & 8 & 0.003 \\
\hline 64 & {$[0.01,0.07]$} & 0.04 & 2 & 0.001 & 8 & 0.003 \\
\hline 96 & {$[0.01,0.07]$} & 0.04 & 2 & 0.003 & 8 & 0.007 \\
\hline 128 & {$[0.01,0.06]$} & 0.05 & 2 & 0.004 & 8 & 0.014 \\
\hline 160 & {$[0.01,0.06]$} & 0.05 & 2 & 0.007 & 9 & 0.021 \\
\hline 192 & {$[0.01,0.07]$} & 0.04 & 2 & 0.011 & 9 & 0.030 \\
\hline 384 & {$[0.01,0.08]$} & 0.05 & 2 & 0.056 & 9 & 0.149 \\
\hline
\end{tabular}

Table 5.3: Results for Example 5.2.

\begin{tabular}{|c|c|c|c|c|}
\hline & \multicolumn{3}{|c|}{ MHSS } & MHSS-base \\
\hline$n$ & $\alpha^{t}$ & IT & CPU & CPU \\
\hline 48 & 0.26 & 431 & 0.023 & 0.023 \\
\hline 64 & 0.20 & 562 & 0.043 & 0.034 \\
\hline 96 & 0.14 & 813 & 0.117 & 0.088 \\
\hline 128 & 0.11 & 1060 & 0.243 & 0.167 \\
\hline 160 & 0.09 & 1289 & 0.434 & 0.287 \\
\hline 192 & 0.07 & 1534 & 0.685 & 0.402 \\
\hline 384 & 0.04 & 2732 & 8.071 & 2.310 \\
\hline
\end{tabular}

Example 5.2. The generating function is given by

$$
f(x)=x^{2}+\imath x^{4} .
$$

The function of Example 5.2 generates a special Toeplitz matrix which has the structure shown in (4.1). That is to say, the real and the imaginary parts of the Toeplitz matrix gen- 
erated in Example 5.2 are symmetric Toeplitz matrices, too. We use MHSS and MHSS-based structured iteration methods to solve the linear system yielded in Example 5.2. According to our tests, the experimentally found optimal parameters $\alpha^{t}$ and the number of iteration steps for MHSS and for MHSS-based structured iteration methods are the same.

In Table 5.3, we list the experimentally found optimal parameters $\alpha^{t}$, and IT and CPU, with respect to different $n$. The results in Table 5.3 show that the MHSS-based structured iteration method costs less CPU time than the MHSS iteration method with respect to different $n$.

Acknowledgments. The work was supported by State Key Laboratory of Scientific/Engineering Computing, Chinese Academy of Sciences; The International Science and Technology Cooperation Program of China under Grant 2010DFA14700; The Natural Science Foundation of China (NSFC) under Grant 11071192, P.R. China.

\section{References}

[1] O. Axelsson, Iterative Solution Methods, Cambridge University Press, 1996.

[2] O. Axelsson and A. Kucherov, Real valued iterative methods for solving complex symmetric linear systems, Numer. Linear Algebra Appl., 7 (2000), 197-218.

[3] O. Axelsson, Z.Z. Bai and S.X. Qiu, A class of nested iteration schemes for linear systems with a coefficient matrix with a dominant positive definite symmetric part, Numer. Algorithms, 35 (2004), 351-372.

[4] Z.Z. Bai, M. Benzi and F. Chen, Modified HSS iteration methods for a class of complex symmetric linear systems, Computing, 87 (2010), 93-111.

[5] Z.Z. Bai and G.H. Golub, Accelerated Hermitian and skew-Hermitian splitting iteration methods for saddle-point problems, IMA J. Numer. Anal., 27 (2007), 1-23.

[6] Z.Z. Bai, G.H. Golub and C.K. Li, Convergence properties of preconditioned Hermitian and skewHermitian splitting methods for non-Hermitian positive semidefinite matrices, Math. Comput., 76 (2007), 287-298.

[7] Z.Z. Bai, G.H. Golub, L.Z. Lu and J.F. Yin, Block triangular and skew-Hermitian splitting methods for positive-definite linear systems, SIAM J. Sci. Comput., 26 (2005), 844-863.

[8] Z.Z. Bai, G.H. Golub and M.K. Ng, Hermitian and skew-Hermitian splitting methods for nonHermitian positive definite linear systems, SIAM J. Matrix Anal. Appl., 24 (2003), 603-626.

[9] Z.Z. Bai, G.H. Golub and J.Y. Pan, Preconditioned Hermitian and skew-Hermitian splitting methods for non-Hermitian positive semidefinite linear systems, Numer. Math., 98 (2004), 1-32.

[10] R.H. Chan and M.K. Ng, Conjugate gradient methods for Toeplitz systems, SIAM Rev., 38 (1996), 427-482.

[11] F. Chen and Y.L. Jiang, On HSS and AHSS iteration methods for positive definite Toeplitz linear systems, J. Comput. Appl. Math., 234 (2010), 2432-2440.

[12] G. Heinig and K. Rost, Fast algorithms for Toeplitz and Hankel matrices, Linear Algebra Appl., 435 (2011), 1-59.

[13] C.Q. Gu and Z.L. Tian, On the HSS iteration methods for positive definite Toeplitz linear systems, J. Comput. Appl. Math., 224 (2009), 709-718.

[14] Y.L. Jiang, R.M.M. Chen and O. Wing, Improving convergence performance of relaxation-based transient analysis by matrix splitting in circuit simulation, IEEE Trans. Circuits Systems: Part I, 48 (2001), 769-780.

[15] Y.L. Jiang, Y.W. Liu, K.K. Mei and R.M.M. Chen, A new iterative technique for large and dense linear systems from the MEI method in electromagnetics, Appl. Math. Comput., 139 (2003), 157-163. 